\title{
Comparison of Arm-Trunk Movement between Complete Paraplegic and Able- Bodied Subjects during Circle Drawing
}

\author{
Guan-Shuo Pan ${ }^{1,2}$, Tung-Wu Lu ${ }^{3}$ and Kwan-Hwa Lin $2,4^{*}$ \\ ${ }^{1}$ Division of Physical Therapy, Department of Physical Medicine and Rehabilitation, National Taiwan University Hospital, Taipei, Taiwan \\ ${ }^{2}$ School and Graduate Institute of Physical Therapy, College of Medicine, National Taiwan University, Taipei, Taiwan \\ 3 Institute of Biomedical Engineering, National Taiwan University, Taipei, Taiwan \\ ${ }^{4}$ Department of Physical Therapy, Tzu Chi University, Hualien, Taiwan
}

"Corresponding author: Kwan-Hwa Lin, Department of Physical Therapy, Tzu Chi University, No.701, Sec. 3, Jhongyang Rd., Hualien City, Hualien County 97004, Taiwan (R.O.C), Tel: 886-3-8461505; Fax: 886-3-8573962; E-mail: khlin@ntu.edu.tw

Rec date: April 05, 2014; Acc date: April 29, 2014; Pub date: May 03, 2014

Copyright: @ 2014 Pan GS, et al. This is an open-access article distributed under the terms of the Creative Commons Attribution License, which permits unrestricted use, distribution, and reproduction in any medium, provided the original author and source are credited.

\begin{abstract}
Study design: This study was a cross sectional, controlled trial.

Objectives: To examine the performance of repetitive circle drawing on dynamic sitting balance between subject with spinal cord injury $(\mathrm{SCl})$ and age-matched able-bodied $(\mathrm{AB})$ adults.
\end{abstract}

Summary of background data: Previous studies showed that the dynamic sitting balance is impaired in SCl. However, the arm-trunk coordinated movement for different directions in seated SCl has not been examined yet.

Methods: Twelve subjects with complete T7-T12 thoracic cord injury (mean age: $36.3 \pm 3.0$ years) and 12 agematched $A B$ adults were recruited. Subjects performed 10 repetitive circle drawing at seated position. The threedimensional motion system (Vicon) was used to measure shoulder, trunk and pelvic angles at sitting position.

Results: The $\mathrm{SCl}$ group displayed an arm-trunk movement with a significantly larger shoulder adduction/ abduction angle $(p<0.001)$, but less trunk flexion/extension, pelvic anterior/posterior tilt and pelvic rotation angles than $A B$ controls $(p<0.05, p<0.001$, and $p<0.001$, respectively).

Conclusions: The small circle drawing is feasible to detect the compensatory movement of shoulder for the impairment of trunk and pelvic control in thoracic SCl. Furthermore, the assistance and guidance of trunk and pelvic movements is important for the arm-trunk coordinated movement in thoracic SCl.

Keywords: Spinal cord injury; Dynamic sitting balance; Arm-trunk motion

\section{Introduction}

Previous studies indicate that the dynamic sitting balance is essential for daily activities in subjects with Spinal Cord Injury (SCI) $[1,2]$. Seelen and Vuurman [3] and Seelen et al. [4] used a bimanual forward reaching movement to study the postural control in ablebodied (AB) subjects, and SCI subjects with high level (T2-T8) lesion and low level (T9-T12) lesion. They found that high thoracic SCI subjects use the cranial parts of thoracic extensors, latissimus dorsi, trapezius muscle, pectoralis major and the serratus anterior to control their sitting balance. Furthermore, the use of alternative postural muscles could only partly reduce functional disability regarding balance control in thoracic SCI subjects. Thus, a trade-off between spinal stability and upper extremity task performance might be made by the thoracic injured SCI subjects.

Individuals with complete thoracic SCI may sit with kyphotic posture and posteriorly tilted pelvis due to the loss of voluntary trunk control [5], and this may reduce the stability for daily activities in seated position [1,2]. The kinematic data of joint angles of shoulder and trunk for proprioceptive inputs might be different between SCI and $\mathrm{AB}$ subjects during seated arm-trunk movement. However, the joint motion of shoulder, trunk and pelvis has not been well examined yet.

The repetitive circle drawing can be used to assess the control and production of timed and coordinated movements for skilled motor behavior [6]. In the study of Fleury et al. [7], they allow the seated children to draw a $10-\mathrm{cm}$ circle repetitively on the tablet to check the timing variability. However, the repetitive circle drawing may require the good arm-trunk control. Furthermore, the core muscles around the thoracic-lumbo-pelvic region, including abdominal muscles and paraspinal muscles are important for thoracic-lumbo-pelvic stability during unstable sitting and functional activity $[8,9]$. Therefore, the purpose of this study was to compare the arm and trunk control between $\mathrm{SCI}$ subjects and $\mathrm{AB}$ subjects during repetitive circle drawing. We hypothesized that the movement angles of shoulder, trunk and pelvis in SCI patients would be different from $\mathrm{AB}$ controls during repetitive circle drawing in the seated position. 
Page 2 of 5

\section{Method}

\section{Subjects}

Twelve individuals who had been diagnosed as complete spinal cord injury (i.e., ASIA impairment scale A) [10,11] at thoracic levels (T7T12) were recruited. They were recruited from the Department of Rehabilitation and Medicine in National Taiwan University Hospital (NTUH), Taipei, Taiwan, and The Potential Development Center for Spinal Cord Sufferers, Taoyuan, Taiwan. There were 12 healthy agematched control subjects recruited from community. The inclusive criteria were: (1) having a defined complete SCI diagnosis corresponding to ASIA impairment scale A, (2) post-SCI for more than 1 year, (3) Without spasticity that would interfere with transfer or sitting balance, (4) being able to sit more than 5 minutes on the chair independently, (5) can follow the instructions or orders, (6) right hand dominant subjects. The exclusive criteria were: (1) any other neurological problem, (2) any orthopedic problems that cause limitation of upper extremity functions, or (3) other systemic infections. The consent form which was validated by the ethic committee of National Taiwan University was signed by each participant.

\section{Clinical assessments}

All participants were examined by the American Spinal Injury Association (ASIA) neurological classification for impairment Scale $[10,11]$. The total motor score of ASIA is 100 with 50 for bilateral upper extremities and 50 for bilateral lower extremities [11]. The total sensory score of ASIA score is 112 with 56 respectively allocated to both sides of the body [11]. The arm length from acromion to third metacarpal head was measured by the yardstick. The SCI patient's muscle strengths by manual muscle testing of lower extremities were rated as 0 (ie., totally paralyzed), and were rated as 5 (i.e., normal) of upper extremities.

\section{Procedure}

The task was a repetitive circle drawing by using the trunk and arm to perform arm-trunk coordinated movement at seated position. There was a plain paper $\left(90 \times 90 \mathrm{~cm}^{2}\right)$ on a height adjustable desk and a diameter $8-12 \mathrm{~cm}$ circle (i.e., being $10 \%$ of the subject's arm length from acromion to third metacarpal head) was drawn on the paper. A 1-cm-width circular track was marked for circular tracing, and the center of the circle was on the sagittal plane of the mid-point of sternum and at the distance of straight-arm length (i.e., the distance from sternum to the center point $=$ arm length). The subject grasped a cylinder with the tip on top of the circular track about $2 \mathrm{~cm}$-distance. A laser switch with on-off signals for each circle was placed at the right-hand side of the circle and at the distance of the straight arm length. The participants were allowed to practice several times to keep elbow as straight as possible (with a supporting splint) while drawing the circles counterclockwisely (i.e., from right to left) at self-selected speed, and they were allowed to use the combined trunk and arm coordinated movements to accomplish the repetitive circle drawing. The participants performed 2 sets of repetitive circle drawings counter clockwisely with 10 repetitive circles per set. Ten minutes rest was allowed between 2 sets of trials.

\section{Instruments and data collection}

Three-dimensional marker trajectory data were measured using a 5camera Vicon motion analysis system at a sampling rate of $120 \mathrm{~Hz}$. Eighteen passively reflective markers were attached to each subject's bony landmarks in order to track the motion of the body segments, including the head (bilateral front the ear), trunk (C7, T8, sternum, xyphoid process, shoulder), right lateral and medial epicondyles, the wrist and bilateral 3rd metacarpal bones, bilateral ASIS, bilateral PSIS and 10 technique reflective markers were located on dominated upper extremity and bilateral iliac crests (Figure 1). Totally, 41 passive reflective markers were put on the anatomical landmark of subjects according to the Helen Hayes Marker-set model, as well as chair, table and cylinder. Reflective markers were placed on the four corners of the drawing paper, the center of a circle, top of cylinder and 4 corners of the chair to locate them spatially (Figure 2).
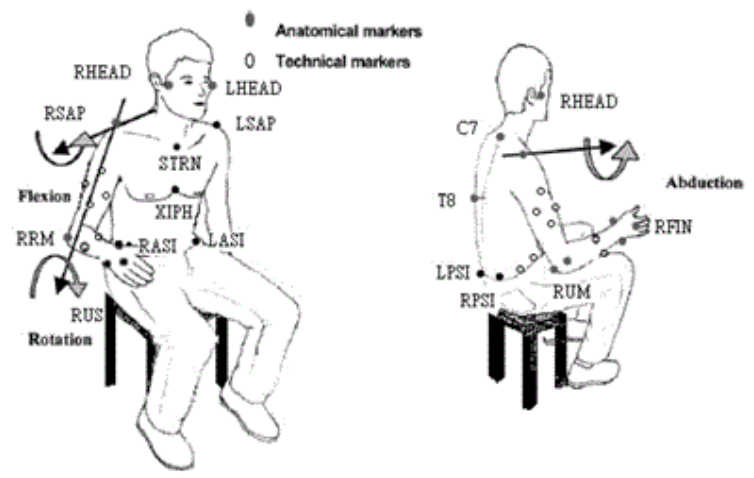

Figure 1: Marker placements in the study (A) Anterior view (B) Right lateral view

\section{Data Analysis}

The variables collected was measured by Vicon motion analysis system software (Workstation ed. 4.6), and were analyzed by the program of Matlab 7.0, to determine the joint angles of arm and trunk. The joint angles were determined from the body segments in reference to the global coordinate system. The axis of the upper arm segment was defined as the line joining right acromion and the mid-point between lateral-medial epicondyles and acromion to define the abduction and adduction of shoulder relative to the trunk segment. An axis joining the $\mathrm{C} 7$ and the mid-point between the two posterior superior iliac spines (PSIS's) was used to define the forward and backward bending of the truncal spine segment relative to the vertical line. The pelvic axis was defined as the line connecting the anterior superior iliac spine (ASIS) and PSIS, and was used to describe the pelvic tilting relative to the hip horizontal plane. The joint angles were calculated by the Euler equations for a four-segment rigid-body biomechanical model [12] to obtain the kinematic data. The variables we used in this study were listed as follows. The shoulder angle described the orientation of the humerus in relation to the trunk. The trunk angle described the orientation of the thorax in relation to the pelvis. 


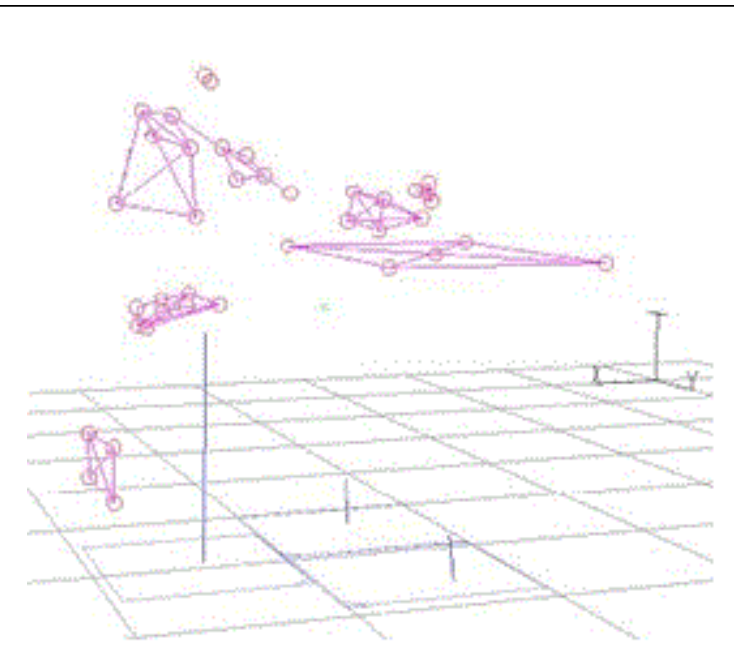

Figure 2: The stick diagram and set-up for motion analysis and center of pressure detection (i.e., upward vertical lines). Three force plates were placed separately under the chair and the feet of subject. The diagram indicates that a subject seats on a chair with arm extension and holding a cylinder by hand. The cylinder (with three markers) is placed on top of a starting switch at the right-hand side of the square table (i.e., with four markers at the corner of the squared drawing paper).

\section{Statistical analysis}

The kinematic data were collected by Vicon motion analysis system software (Workstation ed. 4.6), and would be analyzed by the program of Matlab 7.0. Then, all the data were stored analyzed by SPSS 16.0. The data were presented as mean \pm standard error (SE). KolmogorovSmirnov test (KS-test) and Mauchly's test were used to test the normality and homogeneity of variance respectively. Results were presented as mean values \pm SEM. Independent-samples $T$ test was used for detecting differences between groups. The a was set at 0.05 and $\mathrm{p}<0.05$ would be significant.

\section{Results}

\section{Characteristics of participants}

The demographic information and basic data of $12 \mathrm{AB}$ subjects and 12 complete thoracic SCI (T7-T12) are shown in Table 1, and there are no significant differences in age, height and weight between two groups. The subjects are injured at least one year. The mean motor score of whole body is 50 and the score for lower extremities is ranged from to 0 to 2 . The mean sensory scores of light touch and pin prick are about $70 \%$ of normal.

\section{The joint angle}

The typical examples of the joint angle parameters of a $\mathrm{AB}$ subject and a T10 completely injured SCI are shown in Figure 3 . The X-axis is the normalized time movement (\%), and the $\mathrm{Y}$-axis is the joint angles (degree) for shoulder (curved line), trunk (dashed bar line) and pelvis (dot). The qualitative plots indicate that the pattern of motion is similar, but the movement magnitude of shoulder, trunk and pelvis in
SCI is different from AB controls. In Table 2, the shoulder Adduction/ Abduction angles in SCI group were larger than those of $\mathrm{AB}$ group $(\mathrm{p}<0.001)$, but trunk flexion/extension, pelvic anterior tilt/posterior tilt and pelvic rotation angles in SCI group were smaller than those of $\mathrm{AB}$ group ( $\mathrm{p}<0.05, \mathrm{p}<0.001$, and $\mathrm{p}<0.001$, respectively) during arm-trunk circular movement.

\begin{tabular}{|c|c|c|c|c|}
\hline & $\mathrm{SCl}$ & \multicolumn{2}{|c|}{$A B$} & $p$ value \\
\hline & $(n=12)$ & \multicolumn{2}{|c|}{$(n=12)$} & \\
\hline Age $(y)$ & $37.0(3.1)$ & 36.3 & (3.0) & 0.863 \\
\hline Height $(\mathrm{cm})$ & $171.3(1.9)$ & 171.2 & (1.8) & 0.987 \\
\hline Weight (kg) & $70.9(2.8)$ & 70.4 & (2.5) & 0.897 \\
\hline Duration of injury(yrs) & $5.3(2.1)$ & \multicolumn{2}{|c|}{$(-)$} & \\
\hline Level of injury & T7-T12 & \multicolumn{2}{|c|}{$(-)$} & \\
\hline ASIA Impairment Scale & A & \multicolumn{2}{|c|}{$(-)$} & \\
\hline Motor score & $50.3(0.2)$ & \multicolumn{2}{|c|}{$(-)$} & \\
\hline \multicolumn{5}{|l|}{ Sensory score } \\
\hline Light touch & $70.2(3.3)$ & \multicolumn{2}{|c|}{$(-)$} & \\
\hline Pin prink & $69.2(3.0)$ & \multicolumn{2}{|c|}{$(-)$} & \\
\hline \multicolumn{5}{|c|}{$\begin{array}{l}\text { Spinal Cord Injury Association; Impairment scale A: No motor or sensory } \\
\text { preservation below level of injury }\end{array}$} \\
\hline
\end{tabular}

Table 1: Demographic data of spinal cord injured subjects (SCI) and able-bodied controls (AB)

\begin{tabular}{|l|l|l|l|}
\hline & SCI & AB & p value \\
\hline $\begin{array}{l}\text { Shoulder angle } \\
\text { Adduction/Abduction }\end{array}$ & $21.56 \pm 0.63$ & $13.68 \pm 0.73$ & $0.000^{\star *}$ \\
\hline $\begin{array}{l}\text { Shoulder angle } \\
\text { Flexion/Extension }\end{array}$ & $15.20 \pm 0.88$ & $14.79 \pm 1.09$ & 0.772 \\
\hline $\begin{array}{l}\text { Trunk angle } \\
\text { Flexion/Extension }\end{array}$ & $13.14 \pm 0.61$ & $16.11 \pm 0.19$ & $0.012^{*}$ \\
\hline $\begin{array}{l}\text { Trunk angle } \\
\text { Rotation }\end{array}$ & $12.59 \pm 0.58$ & $12.73 \pm 0.51$ & 0.857 \\
\hline $\begin{array}{l}\text { Pelvic Tilt } \\
\text { Anterior tilt/Posterior tilt }\end{array}$ & $10.78 \pm 1.17$ & $22.64 \pm 2.44$ & $0.000^{* *}$ \\
\hline Pelvic Rotation & $2.03 \pm 0.29$ & $5.41 \pm 0.71$ & $0.000^{* *}$ \\
\hline
\end{tabular}

Table 2: Joint angles changes during circle drawing in seated paraplegics (SCI) and able-bodied controls (AB) 
(1) Able-bodied subject
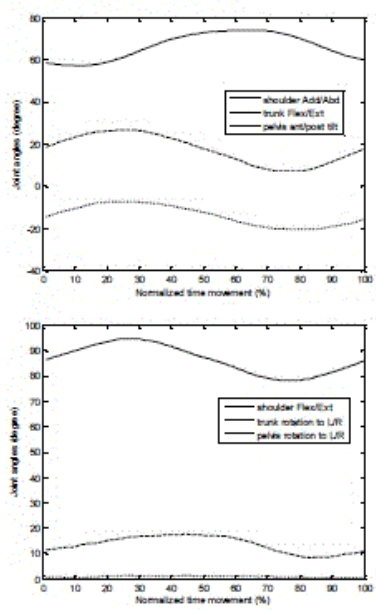

Figure 3: Examples of the joint angles of shoulder and trunk during circle drawing task in an able-bodied subject (1) and a SCI subject (2). Upper panel: shoulder adduction/abduction (curved line), trunk flexion/extension(dashed bar line) and pelvic anterior/ posterior tilt (dot). Lower panel: shoulder flexion/extension (curved line), trunk rotation to $\mathrm{L} / \mathrm{R}$ (dashed bar line) and pelvic rotation to $\mathrm{L} / \mathrm{R}(\mathrm{dot})$.

\section{Discussion}

This is the first study to examine the arm-trunk control during repetitive circle drawing in subjects with complete paraplegic subjects. The SCI individuals used more shoulder motion to compensate the impairment of trunk and pelvic motion. These findings suggested that the SCI subjects used more shoulder movement to achieve arm circle drawing than trunk and pelvic movements because they had to stabilize their trunk to avoid falling. Previous studies of arm motions in SCI subjects had shown that kinematic features were generally similar to non-injured subjects except for minor changes, such as increased scapula winging and slower speeds $[13,14]$.

Previous study had shown that both the paraspinal and the abdominal muscles displayed considerable myoelectric activity modulated in bursts and pauses in normal subjects during arm movements. Further, there was very low baseline activity in abdominal muscles, but considerable activity in the paraspinal muscles [15]. However, thoracic SCI subjects try to compensate for the loss of erector spinae (ES) activity by increased use of the latissimus dorsi, the ascending part of the trapezius, the sternocostal head of the pectoralis major and the serratus anterior $[15,16]$. Therefore, the SCI subjects may change their muscle pattern to achieve arm-trunk coordinated movement. However, present study indicates that it is possible that a trade-off between spinal stability and upper extremity task performance is made by the SCI subjects.

\section{Clinical Application}

It is known that many factors contributed to sitting balance deterioration in the SCI subjects and place them at risk of falling. We found the decrements in the trunk-pelvis control of thoracic SCI adults in repetitive circle drawing. The compensatory arm-trunk movement with larger shoulder displacement and smaller pelvic movement in thoracic SCI may reduce the participation of trunkpelvic muscles. Thus, these results highlight the importance of postural muscle reeducation for arm-trunk coordination and the awareness of the postural feedback (somatosensory feedback) in challenging task conditions. However, this arm-trunk drawing movement may be cautious in acute and subacute subjects, especially with spinal pain at thoraco-lumbar region.

\section{Advantages and Limitations}

The circle drawing requires the eye-arm-trunk coordinated movement, and it may be involved in the daily tasks, such as: controlling the wheel of the car-driving, mopping or cleaning the round table, or controlling the joystick of the video games etc. Therefore, the arm-trunk circle drawing can be the assessment of dynamic postural control of trunk flexion/extension and rotation in different directions. In this study, the participants did not complain discomfort in repetitive circle drawing. However, future study may try to monitor the diameter and the number of circle drawing to check if it is sensitive to reflect the arm-trunk control deficit without producing discomfort.

\section{Conclusion}

The repetitive drawing movement with small circles is feasible for the assessment of arm-trunk control in SCI patients. The dynamic trunk stability in SCI subjects is different from the healthy subjects during arm-trunk circle drawing task. Compared to healthy subjects the thoracic SCI subjects used more shoulder motion to compensate the insufficiency of trunk and pelvic motions in the challenging task. Thus, the thoracic SCI subjects may improve the arm-trunk coordinated movement by the assistance or guidance of the trunk and pelvic control from others.

\section{Acknowledgement}

We gratefully thank all the participants. We also appreciate the assistance from research assistant Chia-Cheng Lin. and the master graduates Fung-Chin Chou and Chia-Jea Chang (Institute of Biomedical Engineering, National Taiwan University) for data analysis. This study was supported by the grant from National Science Council (94-2314-B-002-021).

\section{References:}

1. Alm M, Gutierrez E, Hultling C, Saraste H (2003) Clinical evaluation of seating in persons with complete thoracic spinal cord injury. Spinal Cord 41: 563-571.

2. Minkel JL (2000) Seating and mobility considerations for people with spinal cord injury. Phys Ther 80: 701-709.

3. Seelen HA, Vuurman EF (1991) Compensatory muscle activity for sitting posture during upper extremity task performance in paraplegic persons. Scand J Rehabil Med 23: 89-96.

4. Seelen HA, Potten YJ, Huson A, Spaans F, Reulen JP (1997) Impaired balance control in paraplegic subjects. J Electromyogr Kinesiol 7: 149-160.

5. Sprigle S, Maurer C, Holowka M (2007) Development of valid and reliable measures of postural stability. J Spinal Cord Med 30: 40-49.

6. Zelaznik HN, Spencer RM, Doffin JG (2000) Temporal precision in tapping and circle drawing movements at preferred rates is not correlated: Further evidence against timing as a general purpose ability. J Motor Behav 32:193-199. 
Citation: Pan GS, Lu TW, Lin KH (2014) Comparison of Arm-Trunk Movement between Complete Paraplegic and Able-Bodied Subjects during Circle Drawing. J Spine 3: 165. doi:10.4172/2165-7939.1000165

Page 5 of 5

7. Fleury A, Kushki A, Tanel N, Anagnostou E, Chau T (2013) Statistical persistence and timing characteristics of repetitive circle drawing in children with ASD. Dev Neurorehabil 16: 245-254.

8. Fredericson M, Moore T (2005) Muscular balance, core stability, and injury prevention for middle- and long-distance runners. Phys Med Rehabil Clin N Am 16: 669-689.

9. Spooren AI, Janssen-Potten YJ, Kerckhofs E, Bongers HM, Seelen HA (2011) ToCUEST: a task-oriented client-centered training module to improve upper extremity skilled performance in cervical spinal cordinjured persons. Spinal Cord 49: 1042-1048.

10. Maynard FM Jr, Bracken MB, Creasey G, Ditunno JF Jr, Donovan WH, et al. (1997) International Standards for Neurological and Functional Classification of Spinal Cord Injury. American Spinal Injury Association. Spinal Cord 35: 266-274.

11. Kirshblum SC, Memmo P, Kim N, Campagnolo D, Millis S; American Spinal Injury Association (2002) Comparison of the revised 2000 American Spinal Injury Association classification standards with the 1996 guidelines. Am J Phys Med Rehabil 81: 502-505.
12. Winter D (1990) Biomechanics and motor control of human movement, New York: Wiley.

13. Kulig K, Newsam CJ, Mulroy SJ, Rao S, Gronley JK, et al. (2001) The effect of level of spinal cord injury on shoulder joint kinetics during manual wheelchair propulsion. Clin Biomech (Bristol, Avon) 16: 744-751.

14. Reft J, Hasan Z (2002) Trajectories of target reaching arm movements in individuals with spinal cord injury: effect of external trunk support. Spinal Cord 40: 186-191.

15. Seelen HA, Potten YJ, Drukker J, Reulen JP, Pons C (1998) Development of new muscle synergies in postural control in spinal cord injured subjects. J Electromyogr Kinesiol 8: 23-34.

16. Potten YJ, Seelen HA, Drukker J, Reulen JP, Drost MR (1999) Postural muscle responses in the spinal cord injured persons during forward reaching. Ergonomics 42: 1200-1215. 